\title{
Quark angular momentum and the Sivers asymmetry
}

\author{
Marco Radici* \\ INFN - Pavia \\ E-mail: marco.radiciepv.infn.it
}

\section{Alessandro Bacchetta}

Dipartimento di Fisica - Università di Pavia, and INFN - Pavia

E-mail: alessandro.bacchetta@unipv.it

The determination of quark angular momentum requires the knowledge of the generalized parton distribution $E$ in the forward limit. We assume a connection between this function and the Sivers transverse-momentum distribution, based on model calculations and theoretical considerations. Using this assumption, we show that it is possible to fit at the same time nucleon anomalous magnetic moments and semi-inclusive single-spin asymmetries. This imposes additional constraints on the Sivers function and opens a plausible way to quantifying the quark angular momentum.

Sixth International Conference on Quarks and Nuclear Physics,

April 16-20, 2012

Ecole Polytechnique, Palaiseau, Paris

\footnotetext{
* Speaker.
} 


\section{Introduction}

This talk describes the results of a recent work [1], where we proposed a nonstandard way to constrain the angular momentum $J^{a}$ of a (anti)quark with flavor $a$. In order to do this, we adopted an assumption [2], motivated by model calculations and theoretical considerations, that connects $J^{a}$ to the Sivers transverse-momentum distribution (TMD) [3] measured in semi-inclusive DIS (SIDIS). We showed that this assumption is compatible with existing data, and allows us to derive estimates of $J^{a}$ in fair agreement with other "standard" extractions.

The total angular momentum of a parton $a$ (with $a=q, \bar{q}$ ) at some scale $Q^{2}$ can be computed as a specific moment of generalized parton distribution functions (GPD) [4]

$$
2 J^{a}\left(Q^{2}\right)=\int_{0}^{1} d x x\left(H^{a}\left(x, 0,0 ; Q^{2}\right)+E^{a}\left(x, 0,0 ; Q^{2}\right)\right) .
$$

The GPD $H^{a}\left(x, 0,0 ; Q^{2}\right)$ corresponds to the familiar collinear parton distribution function (PDF) $f_{1}^{a}\left(x ; Q^{2}\right)$. The forward limit of the GPD $E^{a}$ does not correspond to any collinear PDF. It is possible to probe the function $E^{a}$ in experiments, but never in the forward limit. Assumptions are eventually necessary to constrain $E^{a}\left(x, 0,0 ; Q^{2}\right)$. This makes the estimate of $J^{a}$ particularly challenging. The only model-independent constraint is the scale-independent sum rule

$$
\sum_{q} e_{q_{v}} \int_{0}^{1} d x E^{q_{v}}(x, 0,0)=\kappa
$$

where $E^{q_{v}}=E^{q}-E^{\bar{q}}$ and $\kappa$ denotes the anomalous magnetic moment of the parent nucleon.

Denoting the Sivers function by $f_{1 T}^{\perp a}$, we propose this simple relation at a scale $Q_{L}$,

$$
f_{1 T}^{\perp(0) a}\left(x ; Q_{L}^{2}\right)=-L(x) E^{a}\left(x, 0,0 ; Q_{L}^{2}\right),
$$

where $f_{1 T}^{\perp(0) a}$ is the integral of the Sivers function over transverse momentum. This assumption is inspired by theoretical considerations [2] and by results of spectator models [5, 6]. $L(x)$ is a flavor-independent function, representing the effect of the QCD interaction of the outgoing quark with the rest of the nucleon. The name "lensing function" has been proposed by Burkardt to denote $L(x)$ [7]. Computations of the lensing function beyond the single-gluon approximation have been proposed in Ref. [8]. It is likely that in more complex models the above relation is not preserved. Nevertheless, it is useful and interesting to speculate on the consequences of this simple assumption.

The advantage of adopting the Ansatz of Eq. (1.3) is twofold: first, the value of the anomalous magnetic moment fixes the integral of the GPD $E$ and allows us to constrain the valence Sivers function also outside the region where SIDIS data are available; second, our Ansatz allows us to obtain flavor-decomposed information on the $x$-dependence of the GPD $E$ and, ultimately, on quark and antiquark total angular momentum. This is an example of how assuming model-inspired connections between GPD and TMD can lead to powerful outcomes.

\section{Fitting the data}

To analyze SIDIS data, we use the same assumptions adopted in Refs. [9, 10]: we assume a flavor-independent Gaussian transverse-momentum distribution for all inolved TMDs and we neglect the effect of TMD evolution, which has been studied only recently [11]. 
Neglecting $c, b, t$ flavors, we parametrize the Sivers function in the following way:

$$
f_{1 T}^{\perp q_{v}}\left(x, k_{\perp}^{2} ; Q_{0}^{2}\right)=C^{q_{v}} \frac{\sqrt{2 e} M M_{1}}{\pi M_{1}^{2}\left\langle k_{\perp}^{2}\right\rangle}(1-x) f_{1}^{q_{v}}\left(x ; Q_{0}^{2}\right) e^{-k_{\perp}^{2} / M_{1}^{2}} e^{-k_{\perp}^{2} /\left\langle k_{\perp}^{2}\right\rangle} \frac{1-x / \alpha^{q_{v}}}{\left|\alpha^{q_{v}}-1\right|} .
$$

For $\bar{q}$, we use a similar function, excluding the last term. We used $\left\langle k_{\perp}^{2}\right\rangle=0.14 \mathrm{GeV}^{2} . M_{1}$ is a free parameter that determines the transverse-momentum width. We imposed constraints on the parameters $C^{a}$ in order to respect the positivity bound for the Sivers function [12]. We multiply the unpolarized PDF by $(1-x)$ to respect the predicted high- $x$ behavior of the Sivers function [13]. We introduce the free parameter $\alpha^{q_{v}}$ to allow for the presence of a node at $x=\alpha^{q_{v}}$, as suggested in Refs. $[6,14,15]$.

For the lensing function we use the following Ansatz

$$
L(x)=\frac{K}{(1-x)^{\eta}} .
$$

The choice of this form is guided by model calculations [5, 6], by the large- $x$ limit of the GPD $E$ [13], and by the phenomenological analysis of the GPD $E$ proposed in Ref. [16]. We checked $a$ posteriori that there is no violation of the positivity bound on the GPD $E^{q_{\nu}}$.

We performed a combined $\chi^{2}$ fit to the nucleon anomalous magnetic moments (for our present purposes we take $\left.\kappa^{p}=1.793 \pm 0.001, \kappa^{n}=-1.913 \pm 0.001\right)$ and the Sivers asymmetry with identified hadrons from Refs. [17].

We set the gluon Sivers function to zero (its influence through evolution is anyway limited) and we chose $Q_{0}=Q_{L}=1 \mathrm{GeV}$. We fixed $\alpha^{d_{v}, s_{v}}=0$ (no nodes in the valence down and strange Sivers functions). We explored several scenarios characterized by different choices of the parameters related to the strange quark. In all cases, we obtained very good values of $\chi^{2}$ per degree of freedom $\left(\chi^{2} / \mathrm{dof}\right)$, around 1.34 .

All fits lead to a negative Sivers function for $u_{v}$ and large and positive for $d_{v}$, in agreement with previous studies $[18,19,9,10]$. The data are compatible with vanishing sea-quark contributions (with large uncertainties). However, in the $x$ range where data exist, large Sivers functions for $\bar{u}$ and $\bar{d}$ are excluded, as well as large and negative for $\bar{s}$. The Sivers function for $s_{v}$ is essentially unconstrained. The parameter $M_{1}$ is quite stable around $0.34 \mathrm{GeV}$, as well as the strength of the lensing function $K$ around $1.86 \mathrm{GeV}$. The parameter $\eta$ is typically around 0.4 . It turns out $\alpha^{u_{v}} \approx$ 0.78 , so there is little room for a node in the up Sivers function, also because of the constraint imposed by the anomalous magnetic moments.

Our results for the Sivers function are comparable with other extractions [18, 19, 9]. The results for the forward limit of the GPD $E$ are shown in Fig. 1; they also turn out qualitatively similar to available extractions $[16,20]$.

\section{Results for (anti)quark total angular momenta}

Using Eq. (1.1), we can compute the total longitudinal angular momentum carried by each flavor $q$ and $\bar{q}$ at our initial scale $Q_{L}^{2}=1 \mathrm{GeV}^{2}$. Using the standard evolution equations for the angular momentum (at leading order, with 3 flavors only, and $\Lambda_{\mathrm{QCD}}=257 \mathrm{MeV}$ ), we obtain the following results at $Q^{2}=4 \mathrm{GeV}^{2}$ : 


$$
\begin{array}{lll}
J^{u}=0.229 \pm 0.002_{-0.012}^{+0.008}, & J^{d}=-0.007 \pm 0.003_{-0.005}^{+0.020}, & J^{s}=0.006_{-0.006}^{+0.002} \\
J^{\bar{u}}=0.015 \pm 0.003_{-0.000}^{+0.001}, & J^{\bar{d}}=0.022 \pm 0.005_{-0.000}^{+0.001}, & J^{\bar{s}}=0.006_{-0.005}^{+0.000}
\end{array}
$$

The first symmetric error is statistical and related to the errors on the fit parameters, while the second asymmetric error is theoretical and reflects the uncertainty introduced by the other possible scenarios. In the present approach, we cannot include the (probably large) systematic error due to the rigidity of the functional form in Eqs. (2.1) and (2.2). The bias induced by the choice of the functional form may affect in particular the determination of the sea quark angular momenta, since they are not directly constrained by the values of the nucleon anomalous magnetic moments. Our present estimates (at $Q^{2}=4 \mathrm{GeV}^{2}$ ) agree well with other estimates based on the study of the GPD $E[16,20]$ (see Fig. 2). Our study indicates a total contribution to the nucleon spin from quarks and antiquarks of $0.271 \pm 0.007_{-0.028}^{+0.032}$, of which $85 \%$ is carried by the up quark.
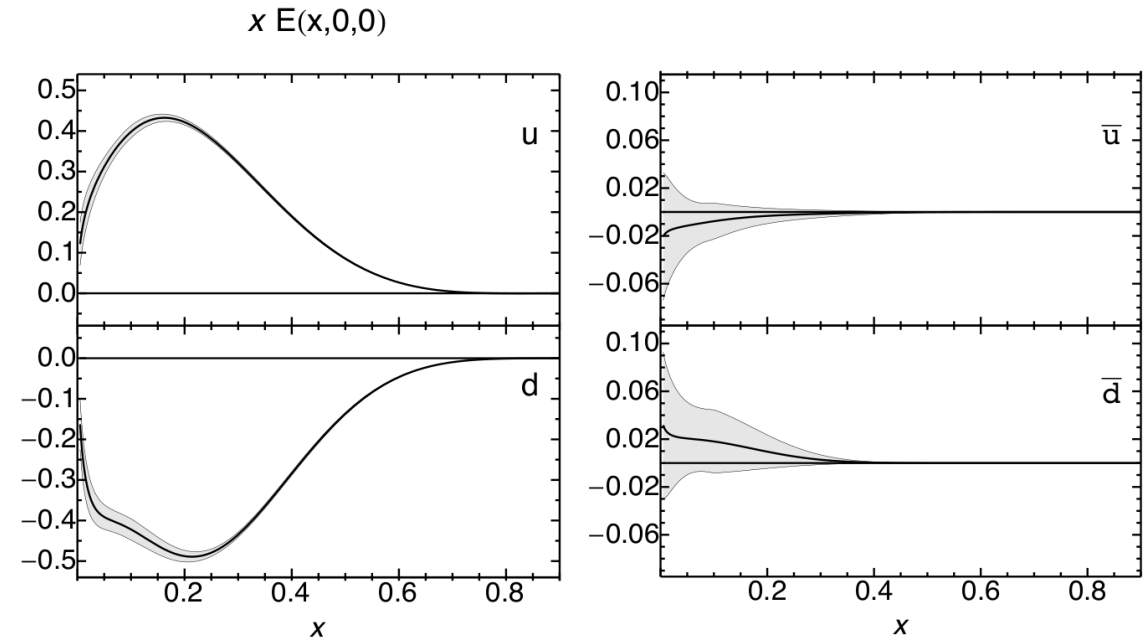

Figure 1: The GPD $x E^{a}\left(x, 0,0 ; Q_{L}^{2}\right)$ as a function of $x$ at the scale $Q_{L}^{2}=1 \mathrm{GeV}^{2}$ for $a=u, d$ (left) and $\bar{u}, \bar{d}$ (right), respectively. The uncertainty bands are produced by statistical errors on the fit parameters.

Our approach can be used also to estimate the size of the total angular momentum carried by the gluons. In this case, we expect the lensing function to be different from that of the quarks. However, our extraction leaves little room for a nonzero gluon Sivers function, since the quark Sivers function already saturate the so-called Burkardt sum rule [23]. If the Sivers function of the gluons is zero, our reasoning allows us to conclude that $E^{g}$ is also zero, independent of the details of the lensing function. This would lead to a value of $J^{g}=0.215$ at $4 \mathrm{GeV}^{2}$. However, these considerations are strongly affected by the uncertainties on the sea-quark Sivers functions outside the $x$ range where data exists. Direct measurements of the sea-quark and gluon Sivers functions are therefore highly necessary.

At this point, we add a remark on the effect of TMD evolution on the Sivers function. The discussions in Refs. [11] suggest that the inclusion of TMD-evolution effects might lead to larger 


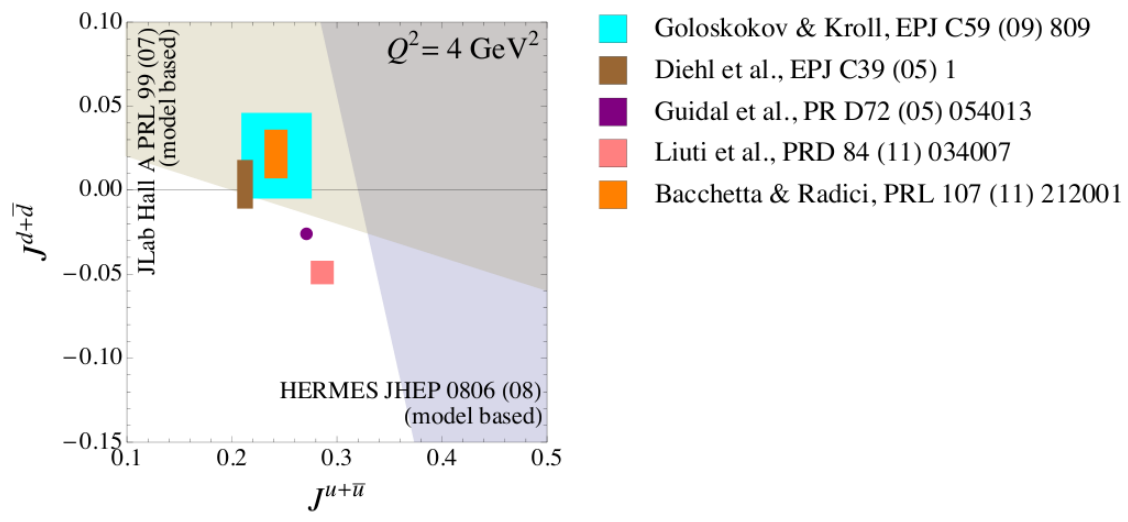

Figure 2: The results of our determination of $J^{u+\bar{u}}$ and $J^{d+\bar{d}}$ compared with other estimates based on GPD extractions from Refs. [16, 20] and measurements of Refs. [21, 22].

values of the Sivers function at the starting scale $Q_{0}^{2}$. If this were the case, we would need to compensate the effect by a smaller size of the lensing function in order to have an agreement with the anomalous magnetic moments. However, this will have a negligible net effect on the results for $J^{a}$.

\section{References}

[1] A. Bacchetta and M. Radici, Phys. Rev. Lett. 107 (2011) 212001.

[2] M. Burkardt, Phys. Rev. D66 (2002) 114005.

[3] D. W. Sivers, Phys. Rev. D41 (1990) 83.

[4] X. Ji, Phys. Rev. Lett. 78 (1997) 610.

[5] M. Burkardt and D. S. Hwang, Phys. Rev. D69 (2004) 074032; Z. Lu and I. Schmidt, Phys. Rev. D75 (2007) 073008; S. Meissner, A. Metz, and K. Goeke, Phys. Rev. D76 (2007) 034002; A. Bacchetta, M. Radici, F. Conti, and M. Guagnelli, Eur. Phys. J. A45 (2010) 373.

[6] A. Bacchetta, F. Conti, and M. Radici, Phys. Rev. D78 (2008) 074010.

[7] M. Burkardt, Nucl. Phys. A735 (2004) 185.

[8] L. Gamberg and M. Schlegel, Phys. Lett. B685 (2010) 95.

[9] S. Arnold et al., arXiv:0805.2137 [hep-ph].

[10] M. Anselmino et al., arXiv:1107.4446 [hep-ph].

[11] S. M. Aybat, J. C. Collins, J.-W. Qiu, and T. C. Rogers, Phys. Rev. D85 (2012) 034043; S. Aybat, A. Prokudin, and T. C. Rogers, arXiv:1112.4423 [hep-ph]; M. Anselmino, M. Boglione, and S. Melis, arXiv:1204.1239 [hep-ph].

[12] A. Bacchetta, M. Boglione, A. Henneman, and P. J. Mulders, Phys. Rev. Lett. 85 (2000) 712.

[13] S. J. Brodsky and F. Yuan, Phys. Rev. D74 (2006) 094018.

[14] Z.-B. Kang and A. Prokudin, Phys. Rev. D85 (2012) 074008. 
[15] D. Boer, arXiv:1105.2543 [hep-ph].

[16] M. Guidal, M. V. Polyakov, A. V. Radyushkin, and M. Vanderhaeghen, Phys. Rev. D72 (2005) 054013.

[17] A. Airapetian et al., Phys. Rev. Lett. 103 (2009) 152002; M. Alekseev et al., Phys. Lett. B673 (2009) 127; X. Qian et al., Phys. Rev. Lett. 107 (2011) 072003.

[18] W. Vogelsang and F. Yuan, Phys. Rev. D72 (2005) 054028.

[19] M. Anselmino et al., Eur. Phys. J. A39 (2009) 89.

[20] M. Diehl, T. Feldmann, R. Jakob, and P. Kroll, Eur. Phys. J. C39 (2005) 1; S. Ahmad, H. Honkanen, S. Liuti, and S. K. Taneja, Phys. Rev. D75 (2007) 094003; S. Goloskokov and P. Kroll, Eur. Phys. J. C59 (2009) 809.

[21] M. Mazouz et al., Phys. Rev. Lett. 99 (2007) 242501.

[22] A. Airapetian et al., JHEP 0806 (2008) 066.

[23] M. Burkardt, Phys. Rev. D69 (2004) 091501. 